\title{
STUDY OF THE DIELECTRIC CHARACTERISTICS OF INDUSTRIAL WOOD
}

\author{
Volodymyr Yalechko, PhD; Andrii Lagun, PhD, As.-Prof. ; Oleksandr Mamro, Student \\ Lviv Polytechnic National University, Ukraine, e-mail: andrii.e.lahun@lpnu.ua
}

\begin{abstract}
In the article, we consider the model of pulse signal distortion in the computer sound card channel. The use of such a model allows expanding the possibilities of using computers in the researches of the wood properties in the form of various dielectric characteristics analysis, in particular using the computer sound card channel. This makes it possible to verify the state of the signal propagation channel during the experiment. This experiment researches samples of wood for the presence of defects.

Also is proposed an approach to constructing the distortion model. It allows to expand the possibilities of investigating the parameters of the pulse signal and to verification the state of the channel during the research of the dielectric properties of wood.

To research, a sample of wood using a computer sound card is developed a structured scheme that allows you to analyze the characteristics of pulse signals during the measurement process.

To determine the dielectric parameters of different types of industrial wood is developed the software in the MATLAB environment. Using it is analyzed complex pulse signals that used during the research. In particular, were taken into account different currents of wood polarization and dielectric characteristics.

Key words: Pulse signal; Dissemination channel; Dynamic distortion; High-pass filter; Computer sound card; Wood.
\end{abstract}

\section{Introduction}

Wood has long been important by mankind in particular for construction due to its construction and technological properties. These properties are significant tensile and compressive strength, low thermal conductivity, manufacturability, and good appearance. The current problem is the preservation of forest reserves in Ukraine and the reduction of applying wood in construction. An important reserve of wood savings is the use of waste from sawmilling and woodworking for the manufacture of particleboard, glued structures, heating means for buildings.

Industrial wood has such disadvantages as the heterogeneity of the structure, flammability, and the ability to rot. These disadvantages are avoided by different technical measures. Researchers exploit different measurement methods to determine the characteristics of wood as a dielectric material.

\section{The Current State of the Problem}

Much of the primary information in physical experiments, which is processed by technical means, is analogous. Bandwidth limitation is a common feature of analog signal transmission channels $[1,2]$. It distorts the test signal parameters. Therefore, the study of the distortions ways is an important general technical task, the successful solution of which is necessary for increasing the productivity of many technical devices.

Different authors research many ways of signals transmission and changing their parameters in the distribution channel [3-10]. For technical tests, they are used monochromatic, narrowband, and broadband signals. Pulse rectangular signals belong to the latter type and are simple in shape and non-complicated methods for creation.

\section{The Goal of the Work}

The purpose of the article is to solve the problem of developing a model of dynamic distortions of the pulse signal that occur during investigations of the properties of wood, in particular the presence of defects in it. The parameters of dielectric materials wood are measured exploiting specialized metrological modules digital oscilloscopes, specialized spectrometers, etc. It is proposed to provide measuring of the wood dielectric properties by a computer sound card channel, which significantly increases the speed of the measurement process.

\section{Development of the Model for a Wood} Sample Study

To investigate the response of any automated measurement system are required a test signal generator and system response analysis tools. It is convenient to programmatically set the test signal in digital form, to test the object of study using an analog signal, and to perform the processing of results with numerical methods.

The application of a set of separate specialized devices for the study is associated not only with the accumulation of hardware and financial costs, but also requires the coordination and synchronization of individual nodes, the correct choice of parameters for each testing.

We suggest applying for a computer sound card in the process of measuring wood characteristics. It includes such nodes of input and output of analog signals as amplifiers, digital-to-analog and analog-to-digital converters (DAC, ADC), clock frequency generator [11], as well as automated control systems for these devices. Appliance the specialized metrological modules is limited by their significant cost.

Comprehensive finding functional relationships between the parameters of the object by the computer capabilities assess the degree of the correlation processes that take place in it provides significant advantages in the choice of processing tools, automation of the experiment. These investigations can be attributed to the 
first stage of measurement - the collection of a priori information. The obtained data are important to the development of methods for obtaining a reliable metrological assessment with special measuring instruments.

The proposed approach to building a model of deviations allows not only expanding the possibilities of restoring the parameters of the pulse signal $[12,13]$ but also by the peculiarities of these deviations to verification the state of the channel during research.

To generate a test signal, to receive and process the reaction of the object of investigation, are important different computer components, shown in Fig. 1:

- To perform numerical operations - memory, clock frequency generator $(\mathrm{CFG})$, processor, bus for data communication.

- To perform signal shape conversion operations and analog processing - sound card units: DAC, ADC, amplifiers (AMP1 and AMP2), analog filters (AF1 and AF2).

To optimize the electrical coordination of the computer with the object of investigation are used external devices: sensor, input, and output amplifiers (AMP).

The bandwidth restriction of the sound card in the below part occurs in the high-pass filter (HF) formed by the separating $\mathrm{RC}$ circuits. These filters consist of electrolytic capacitors and the different resistance values of the sample. Due to the characteristics of the formed elements, these filters are characterized by unstable temperature and time parameters.

The bandwidth of external devices is wider than the bandwidth of the sound card, therefore we can ignore the dynamic signal distortion in these nodes. Dynamic signal distortions depend on the settings of the filters, the temperature of the sound card elements, the characteristics of the connected load, and tend to drift. The presence of unpredictable factors of dynamic distortions in the generation channel makes it necessary to verification the shape of the test signal during the studies.

To perform such verification, the voltage of the output amplifier is applied to one of the stereo inputs of the sound card. The signal that appears at this input is a verification signal. The test signal passes through the same nodes, but needs the second stereo input of the sound card, and additionally passes through the sample, the sensor, and the amplifier of the input signal (Fig. 1). The difference between the test signal and the verification determines the characteristics of the object of investigation.

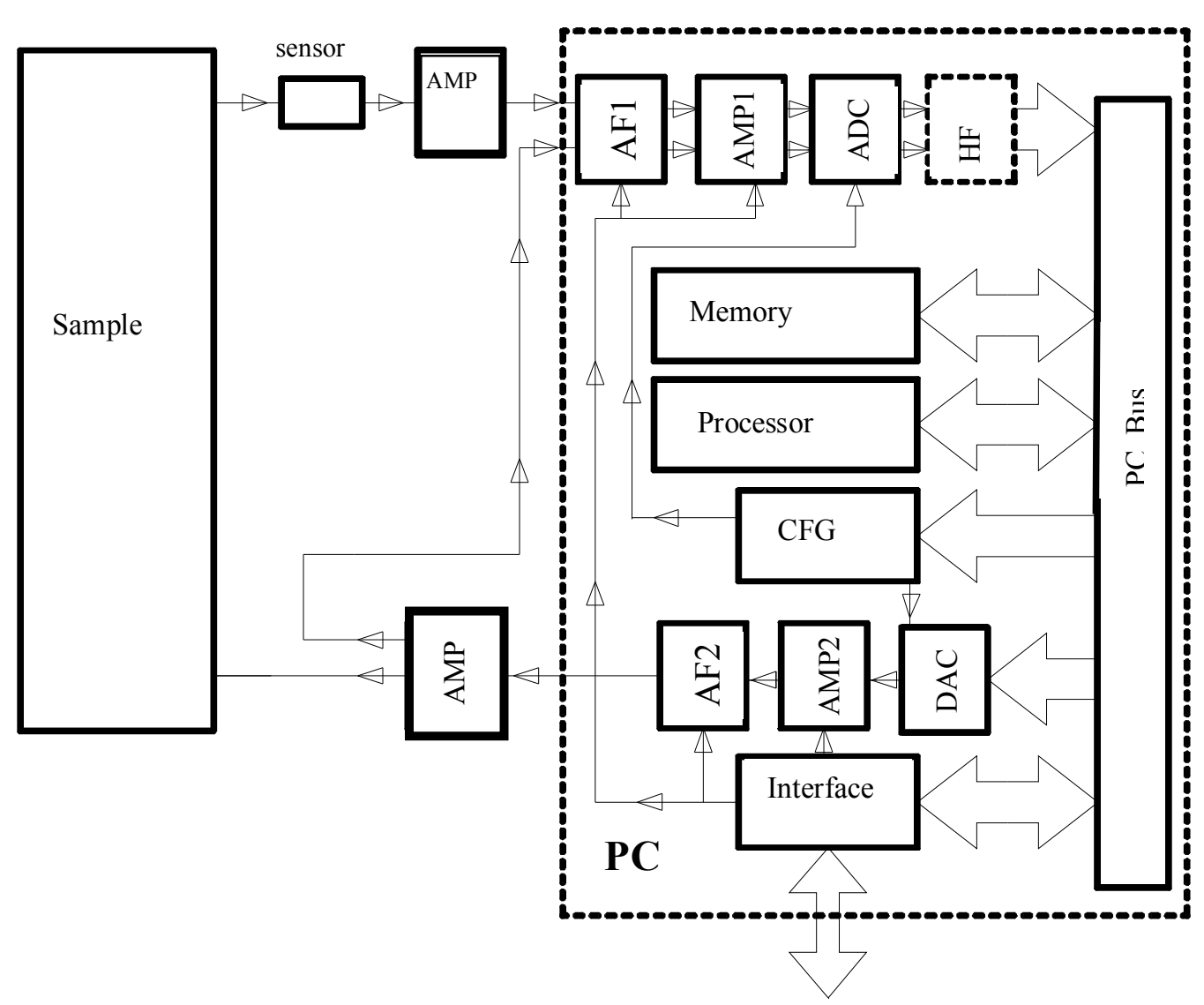

Fig. 1. Structure scheme of a wood sample studies using the computer components 


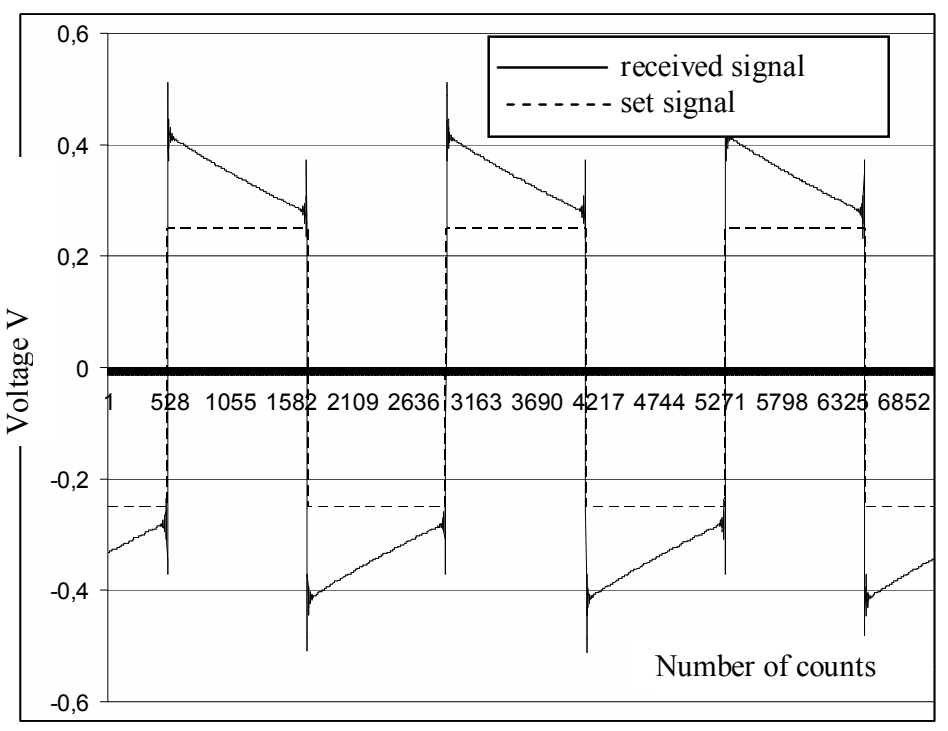

Fig. 2. The signals: set and received forms in the verification channel

\section{Experiments}

To study the properties of wood, we exploit one of the simplest forms of the test pulse signal, which is a meander with a frequency of $80 \mathrm{~Hz}$. The choice of the signal is justified by the lack of a constant component, which cannot be transmitted by a sound card and existing a simple form of dynamic distortions.

Generation of a test signal - meander, with the period of $2 N_{0}$ counts occurs in the form of a digital sequence:

$$
U_{0}(n)=A_{0} \times(-1)^{[n / N 0]},
$$

where $A_{0}$ is the amplitude of the meander; $n$ is the number of counting; $N_{0}$ is the number of counts in the half period of meander, $\left[n / N_{0}\right]$ is the integer part of $n / N_{0}$. There are two forms of signals (Fig. 2) i.e. the signal in digital form and the analog verification signal. Are observed distortions on the received signal: lowfrequency - on the slope of the pulse peak, and highfrequency distortion - in the form of damped oscillations at the beginning and end of the pulses.

After passing the meander is created another sequence corresponding to the verification signal $E(n)$, with a changed shape and with a noise component (Fig. 2):

$$
E(n)=U(n)+U_{n}(n),
$$

where $U(n)$ is a periodic component of the verification signal; $U_{n}(n)$ is the value of the additive noise component. To distinguish the shape of the received verification signal from the noise, $N_{k}$ counts are divided into $K$ integer periods of $2 N_{0}$ counts in each, and depending on the offset of the beginning of the first period $n_{0}$ :

$$
K=\left[\frac{N_{k}-n_{0}}{2 N_{0}}\right],
$$

and the values of the verification signal $E(n)$ were averaged over $K$ periods for each value $m=\left[1,2 N_{0}\right]$ :

$$
\begin{gathered}
Y(m)=\frac{1}{K} \sum_{k=1}^{K} E\left(2 K N_{0}+m\right)=\frac{1}{K} \sum_{k=1}^{K} U\left(2 K N_{0}+m\right)+ \\
+\frac{1}{K} \sum_{k=1}^{K} U_{n}\left(2 K N_{0}+m\right)
\end{gathered}
$$

Approximation of the slope of the top of the verification signal by the exponential curve is carried out by the formula:

$$
U_{c}(i)=A_{c} e^{-i / N c},
$$

where $A_{c}$ is the amplitude, $N_{c}$ is the value of the constant exponent. The approximation parameters $A_{c}$ and $N_{c}$ are determined from the condition of the minimum standard deviation of the approximating curve $U_{a}(i)$ and the average values of the received verification signal $Y_{a v r}(i)$ :

$$
\sigma=\sqrt{\frac{1}{1098} \sum_{k=52}^{1149}\left(Y_{\text {avr }}(i)-A_{c} e^{-\frac{i}{N_{c}}}\right)} \rightarrow \min .
$$

As a result of the approximation, is obtained the values of the meander amplitude and the value of the time constant, which characterizes the distortion of the shape of both the verification and test signals in the sound card. It should be noted that although the formula (6) contains index values greater than 51 , the optimization determines the value of $A_{c}$, which corresponds to equality $i=0$; and the value of $N_{c}$ is not an integer, but real. The selected approximation function and the method of determining its parameters provided in the experiments the value of the multiple determination coefficient of the test pulse peak decline $R_{2} \geq 0.99$.

Computer sound cards to receive stereo signals include two channels. The characteristics of the channels are a high degree of identity, so the methods of compensation for distortion of the verification channel must be effective for the test signal. With the model of the exponential decay of the pulse peak, we can restore the shape of each signal by distributing discrete values of $E(n)$ to the approximation function $U_{a}(n)$ with single 
amplitude when synchronizing the front of the test signal with the beginning of the approximation function:

$$
U_{r}(n)=E(n) / U_{c}\left(\left(N_{0}-n_{0}+n\right) / N_{0}\right)=E(n) / e^{-i / N c},
$$

where $U_{r}(n)$ is the restored form of the test signal, $N_{0}$ is the index of approximation function synchronized with the front of the test signal.

The obtained function $U_{r}(n)$ transmits the pulse front and the damping oscillations of the transient process, which occurred in the low-pass filter, and also the properties of the noise component. A flat vertex of this function is characteristic of a meander-shaped pulse. To verify the conformity of the distortion model in the high-pass filter (HF) through the channel instead of the sample were connected resistors of different denominations. The difference in the value of the resistor affects not only the value of the current perceived by the current sensor. Therefore, occurs difference the time constant of the HF and, accordingly, the values of $A_{c}$ and ${ }_{N c} \mathrm{i}$ the model of the distortion of the pulse peak. With all values of the resistor in the range from 0.47 to $15 \mathrm{Mega}$ Ohms, the restoration of the flat top of the meander occurred to the nearest noise level. Thus, the known shape of the set test signal, the presence of the verification channel of the test signal shape, the selected model, and the method of compensation for distortion of the HF restore the shape of the test signal in the measurement channel.

For fast and convenient determination of model, parameters are developed software in the MATLAB environment. As a result of practical researches is obtained high reliability and accuracy of recovery. Investigations have been carried out with complex pulse signals, including polarization currents of wood and other dielectric characteristics.

\section{Conclusions}

The developed models of signals obtained in real transients of pulse signal propagation do not require structural division of the channel into separate nodes with the determination of their characteristics. Parameters of these models verification the state of the signal transmission channel. That increases the reliability of technical means as well as the reliability of the received results.

The meander test pulse signal and the real pulse signal obtained after sensors for a specific wood sample investigations of woods' samples. We obtain the required dielectric characteristics of wood using only computer hardware, in particular the computer sound card.

The coincidence with the accuracy of the noise level of the indicators of the digital oscilloscope ZET 302 [14] with the counts of the restored sound card signal proves the possibility of computer technique for measurements. So, we have studied the possibilities of computer hardware as an available technologically and metrologically reliable mean for verification of the dielectric properties of materials, in particular wood.

\section{Conflict of interest}

The authors state that there are no financial or other conflicts concerning the current work.

\section{References}

[1] A. Oppenheim, A. Willsky, S. Nawab, Signals and Systems (2nd Edition), Prentice-Hall, 1996. [Online]. Available: https://eee.guc.edu.eg/Courses/Communications/COMM401\%20S ignal $\% 20 \& \% 20$ System $\% 20$ Theory/Alan $\% 20 \mathrm{~V} . \% 20$ Oppenheim, \%20Alan\%20S.\%20Willsky,\%20with\%20S.\%20HamidSignals\%20and\%20Systems-Prentice\%20Hall\%20(1996).pdf

[2] Ye. Polishhuk, M. Dorozhovec, V. Yatsuk, Metrology and measurement technology, Beskids Beat, 2012. [Online]. Available: https://vlp.com.ua/node/8983

[3] V. Suslyaev, T. Kochetkova, G. Dunaevskii, K. Dorozhkin, "Research of dielectric properties of wood at frequencies $0.1 \div 0.5 \mathrm{THz}$ ", 1-2, 2014. DOI: 10.1109/IRMMWTHz.2014.6956102

[4] T. Saeidi, I. Ismail, W. Wen, A. Alhawari, "Ultrawideband elliptical patch antenna for microwave imaging of wood. International Journal of Microwave and Wireless Technologies", 2019. DOI: 11. 10.1017/S1759078719000588

[5] N. Abdurakhimov, A. Lagunov, V. Melehov, "Study of influence of $2.4 \mathrm{GHz}$ electromagnetic waves on electrophysical properties of coniferous trees wood", in Proc. AIP Conference, 2017, 020048. DOI: 10.1063/1.5002945

[6] T. Saeidi, I. Ismail, A. Alhawari, W. Wen, "Nearfield and far-field investigation of miniaturized UWB antenna for imaging of wood", AIP Advances, no.9, 2019, 035232. DOI: $10.1063 / 1.5081762$

[7] S. Tanaka., Y. Fujii, K. Shiraga, Y. Ogawa, "Relation between anisotropic relative permittivity and density of wood evaluated using $\mathrm{THz}$ time-domain transmission spectroscopy", in Proc. 38th IRMMW-THz International Conference, TU14-3, Mainz on the Rhine, Germany, 2013. DOI: 10.1109/IRMMWTHz.2013.6665580

[8] C. Hill, Wood Modification: Chemical, Thermal, and Other Processes, 2006. [Online]. Available: https://download.ebookshelf.de/download/0000/5677/02/L-G-0000567702-

0015270636.pdf

[9] B. Gardiner, J. Barnett, Z. Saranp, J. Gril, The Biology of Reaction Wood, 2014. [Online]. Available: https://ink.springer.com/book/10.1007\%2F978-3-642-108143\# editorsandaffiliations

[10] R. Magagi, M. Bernier, C. Ung, "Analysis of RADARSAT SAR Data Over a Sparse Forest Canopy", IEEE Transactions on Geoscience and Remote Sensing, vol.40, p.13011313, 2002. DOI: 10.1109/TGRS.2002.800235

[11] M. Kabir, W. Daud, K. Khalid, A. Sidek, Equivalent circuit modeling of the dielectric properties of rubber wood at low frequency. Wood and Fiber Science. 32. 2000, [Online]. Available: https://www.srs.fs.usda.gov/pubs/VT_Publications/00t27.pdf

[12] P. Hwei, Theory and Problems of Signals and Systems, McGraw-Hill Companies, USA, 1995. [Online]. Available: http://people.disim.univaq.it/ costanzo.manes/EDU_stuff/Theory\% 20 and $\% 20$ Problems $\% 20$ of $\% 20$ Signals $\% 20 \& \% 20$ Systems_Hsu_S chaum95.pdf

[13] A. Oppenheim, R. Schafer, Discrete-Time Signal Processing (Prentice-Hall Signal Processing Series) 3rd Edition, Pearson, 2009. [Online]. Available: https://azrael.digipen.edu/ MAT321/DiscreteTimeSignalProcessing3.pdf

[14] Digital Oscilloscope ZET 302. Passport. User manual. CJSC "Digital technology and metrology systems", Zelenograd, 2010. [Online]. Available: https://zetlab.com/en/shop/ adc-dac-modules-digital-usb-oscillographs/oscillograph-zet-302/ 